\title{
Erratum: Mercury Monohalides: Suitability for Electron Electric Dipole Moment Searches [Phys. Rev. Lett. 114, 183001 (2015)]
}

V. S. Prasannaa, A. C. Vutha, M. Abe, and B. P. Das

(Received 13 August 2016; published 29 August 2016)

DOI: 10.1103/PhysRevLett.117.109901

In Table I of the manuscript, the basis for iodine has been specified as $8 s, 6 p, 6 d$. It must be $21 s, 15 p, 11 d$. This was a typographic error, and the results (for HgI) given in the manuscript are correct, and is for the correct choice of basis, $21 \mathrm{~s}$, $15 p, 11 d$. 